\title{
QoS Route Optimization Algorithm for the Dynamic Traffic and Network Service
}

\author{
Yanping $\mathrm{Chen}^{1}$ and Yulong $\mathrm{Gao}^{2}$ \\ ${ }^{1}$ Department of Computer and Information Engineering, Harbin University of \\ commerce, Harbin, China \\ ${ }^{2}$ Communication Research Center Harbin Institute of Technology, Harbin, China, \\ Yanping1009@163.com,ylgao@hit.edu.cn
}

\begin{abstract}
Transforming the convolution to the (+,-) in legend domain is proposed in this paper. Based on the transformation, the Legend Transformation of service curve and arrive curve is given in the case of independent cross traffic, and the closed expression of stochastic delay and stochastic backlog is obtained. In the paper, we analyze influence of the dynamic of traffic and network to QoS parameter. Based on the analysis result, the QoS analysis in Legend domain is given, and the upper bound of delay and backlog in Legend domain. For the case of non-independent cross traffic, the relationship expression of different traffic is got. And simulation proves that the route optimization algorithm is correct.
\end{abstract}

Keywords: QoS Route, Legend Transformation, Service Curve, Arrive Curve

\section{Introduction}

To achieve route optimization in dynamic network, we need to model dynamic network firstly. It is assumed that the end-to-end matrix of traffic QoS is delay, the other traffic is called cross traffic ${ }^{[1]}$, the QoS parameters is decided by traffic and service provided by network, and service curve is influenced by cross traffic and the interaction between cross traffic and current traffic. In this paper, the independent situation between current traffic and cross traffic is mainly studied, the QoS route optimization algorithm in the simplest case is proposed simultaneously.

When current traffic is independent of cross traffic, that is, cross traffic doesn't have effect on current traffic, the simplest case is that cross traffic goes through only on network node, the case is shown in figure 1 . In the figure, the dashed line represents the route of cross traffic, the solid line represents the route of traffic $f$. To Study the QoS route problem in the extreme case, we explore the computing problem of QoS route selection using Network Calculus and Legend Transformation.

\section{Network Dynamic Analysis}

From the definition of delay and backlog in Network Calculus, it can be known that delay and backlog is decided by the network service function and traffic function, and the two functions is dynamic, therefore, QoS parameter delay and backlog is dynamic. To analyze the influence factors for QoS parameter, geometric form shown in Figure 1 is taken to analyze the relationships among delay, backlog and traffic function, network node service function.

In time $t$, delay and backlog is respectively the horizon distance and vertical dist ance between arrival curve and service curve. The traffic varies randomly, and the service 
curve provided to the traffic varies too, therefore the delay and backlog changes in real time. For the real time, changing QoS parameter is difficult to use QoS route calculation. But from the analysis above, the upper bound of delay and backlog is determined for some period of time, therefore delay or backlog upper bound in a slot time can be taken as the metric parameters.

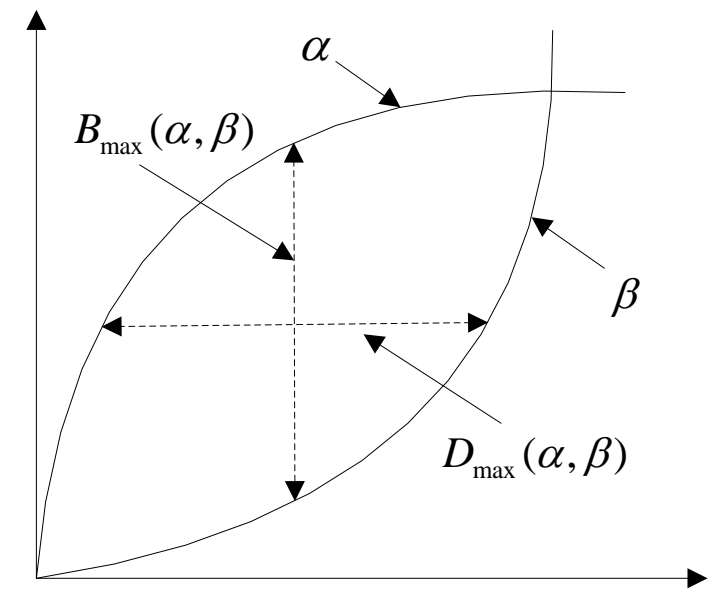

\section{Figure 1. Geometric Representation of Backlog and Delay Bound}

In addition, many factors lead to instability of network. When the network is instable, the delay and backlog parameter is unbound, then QoS route is impossible. Therefore, it is assumed that network is stable, and then the delay and backlog is bound, based on which we study the QoS route optimization, and we take the network topology shown in Figure 2.

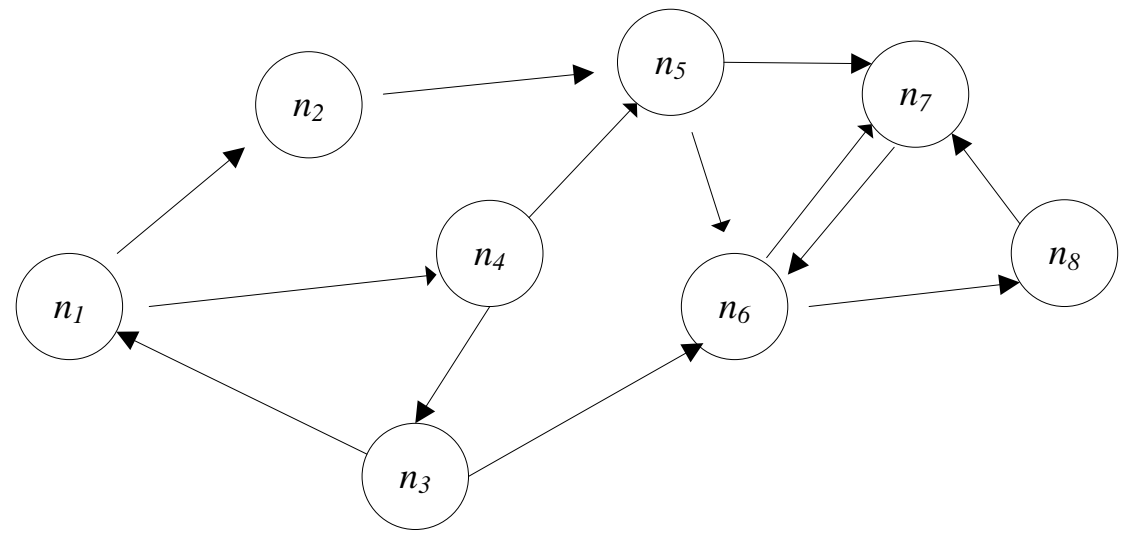

Figure 2. Network Topology

In addition to the stability of network, the difficulty to characterize QoS parameter is the complex relationship between non-independent traffics, especially there is cross traffic for current traffic, this situation occurred when there is cross path for much traffic. Take the Figure 3 as example, assume that the traffic $F_{1}$ go through node $n_{1}$ and $n_{2}$ to node $n_{5}$, in which traffic $F_{3}$ and traffic $F_{1}$ have the same transmission path, 
and the source node of traffic $F_{2}$ is node $n_{1}$ and the end node is $n_{2}$. To analyze the QoS parameter of traffic $F_{1}$ path $\left(n_{1}, n_{2}, n_{5}\right)$, according to the network calculus, first we need to obtain the service curve provided to traffic $F_{1}$ by path $\left(n_{1}, n_{2}, n_{5}\right)$. The most direct calculation method is to use the left service curve theorem to calculate every node service curve provided to traffic $F_{1}$, then according to the convolution, the service curve of path $\left(n_{1}, n_{2}, n_{5}\right)$ provided to traffic $F_{1}$ can be obtained.

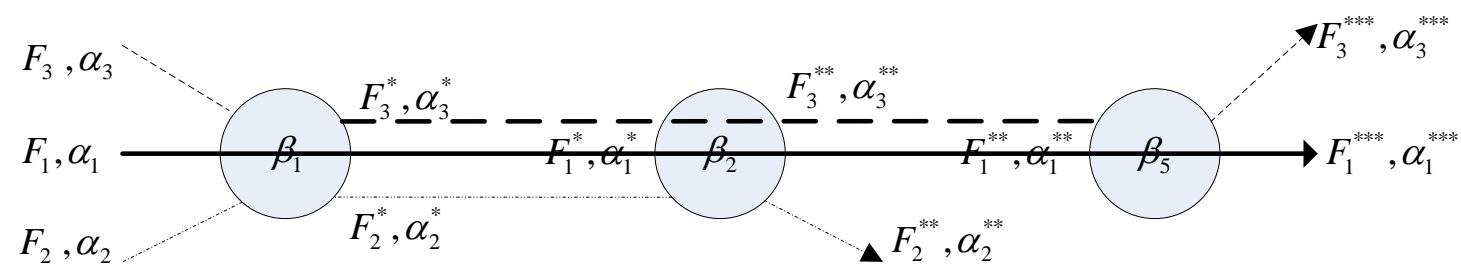

Figure 3. Traffic Flow in Network Node

Take the conditions shown in figure 3 as example, the service curve of path $\left(n_{1}, n_{2}, n_{5}\right)$ provided to $F_{1}$ is

$$
\beta_{\text {path }}^{1}=\left[\beta_{1}-\alpha_{2}-\alpha_{3}\right]^{+} \otimes\left[\beta_{2}-\alpha_{2}^{*}-\alpha_{3}^{*}\right]^{+} \otimes\left[\beta_{3}-\alpha_{3}^{* *}\right]^{+}
$$

Analyzing the path of traffic in figure 3 , we can find that the traffic $F_{3}$ and $F_{1}$ have the same path, and the path of $F_{2}$ is the sub-path of path of $F_{1}$. From the view of subpath, the service curve provided to traffic $F_{1}$ is calculated by

$$
\beta_{\text {path }}^{1}=\left[\left[\left(\beta_{1} \otimes \beta_{2}\right)-\alpha_{2}\right]^{+} \otimes \beta_{3}-\alpha_{3}\right]^{+}
$$

Changing all variables to specific value and comparing the service curve obtained by two calculation method, it can be found that the delay is small. The reason is that when traffic $F_{1}$ converge other traffic, the first network node influent the current traffic severely, the service obtained by the current traffic greatly reduced, however, in subsequent node, the influence by the resource competition in the first node is solved partly. Therefore, the interference coming from other traffic reduced, which is call PMOO(Pay Multiplexing Only Once) .Using the sub-path calculation method for the service and taking account the PMOO into the service curve calculation, better service curve can be obtained.

\section{QoS Analysis in Legend Domain}

To analyze the QoS in Legend domain, assume the arrive curve is concave, and concave function through original point is subadditive, therefore the concave arrive curve is subadditive. Then, concave arrive function can characterize the traffic arrival process. Next, Legend Domain QoS analysis is performed for the concave arrive function and convex service function.

Assume the arrival process of traffic $F$ satisfied the following stochastic service curve

$$
P\{A(t)>A \otimes E(t)+\sigma\} \leq \varepsilon_{E}(\sigma)
$$


And the service curve provide by the network satisfied

$$
P\{D(t)<A \otimes S(t)-\sigma\} \leq \varepsilon_{S}(\sigma)
$$

According to the analysis of section 2, the biggest delay and backlog of traffic $F$ is respectively the horizontal distance and vertical distance. But, the biggest delay and backlog obtained method is based on determined network calculus. According to definition of stochastic delay and backlog, we need to obtain the error function of stochastic definition for getting stochastic QoS parameter of traffic. Next the concave, convex function and Legend Transformation is exploited to get stochastic QoS parameter.

Theorem 5.1(The Backlog Bound in Legend Domain):Known the service curve of network node is $\beta(t)$, and $Q(t)$ represents the backlog of network node in time $t$, $D(t)$ represents the delay of traffic going though in time $t$, its arrive curve is $\alpha(t)$, the Legend Transformation of the arrive curve is

$$
A(s)=\underline{L}_{\alpha}(s)=\inf _{t}[s \cdot t-\alpha(t)]
$$

The Legend Transformation of service curve satisfied

$$
B(s)=\bar{L}_{S}(s)=\sup _{t}[s \cdot t-\beta(t)]
$$

The biggest backlog bound $Q$ in Legend domain satisfied

$$
Q=-\sup _{s \in \square}\{A(s)-B(s)\}
$$

The biggest delay bound $\mathrm{D}$ satisfied

$$
D=B^{\prime}\left(s^{*}\right)-A^{\prime}\left(s^{*}\right)
$$

here $A^{\prime}(s), \quad B^{\prime}(s)$ represent respectively the tangent line of $A(s)$ and $B(s)$, and $A^{\prime}(0)=B^{\prime}(0)$, they tangent in point $s^{*}$.

Next we take the arrive curve as example, and analyze the relationship between the determine network calculus and stochastic network calculus.

Assume the arrive envelope of traffic $E(t)$ is concave, and the service curve $S(t)$ is convex. We can do the Legend Transformation for the $E(t)$ and $S(t)$ respectively

$$
\begin{gathered}
\bar{L}_{E}(c)=\sup _{t \geq 0}\{c t-E(t)\} \\
\underline{L}_{S}(c)=\inf _{t \geq 0}\{c t-S(t)\}
\end{gathered}
$$

Known that the service curve satisfied $\left\langle S(t), \varepsilon_{S}(\sigma)\right\rangle$, and scheduling strategy is FIFO, the arrive curve of traffic satisfied $\left\langle E(t), \varepsilon_{E}(\sigma)\right\rangle, \forall c, \sigma_{E}, \sigma_{S} \geq 0$, then the stochastic backlog $B$ satisfied

$$
P\left\{B>\bar{L}_{E}(c)+\underline{L}_{S}(c)+\sigma_{E}+\sigma_{S}\right\} \leq \varepsilon_{E}\left(\sigma_{E}\right)+\varepsilon_{S}\left(\sigma_{S}\right)
$$


And the delay $W$ satisfied

$$
P\left\{W>\left(\bar{L}_{E}(c)+\underline{L}_{S}(c)+\sigma_{E}+\sigma_{S}\right) / c\right\} \leq \varepsilon_{E}\left(\sigma_{E}\right)+\varepsilon_{S}\left(\sigma_{S}\right)
$$

\section{Network Model}

First of all, we study the case of cross traffic independent each other, and we know that the current traffic is F. Take the simplest case as example, each cross traffic goes through only one network node, that is the cross traffic $f_{i}$ goes through only one node of path $p_{i}$, and we can get that the arrive curve of traffic $f_{i}$ is $\left\langle\alpha_{i}, \varepsilon_{i}\right\rangle$, which is shown in Figure 4.

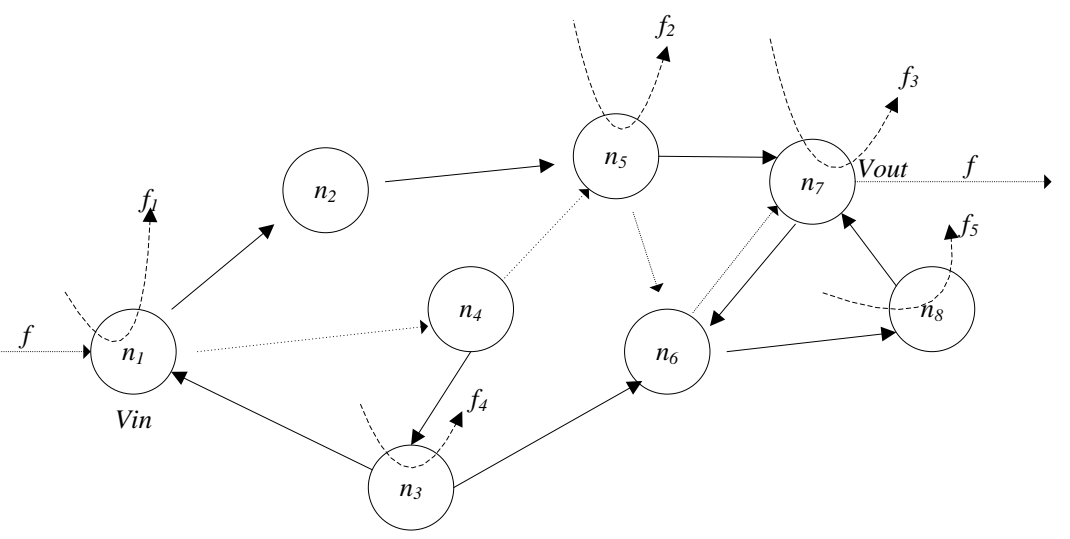

Figure 4. Network Model for Independent Cross Flow

The network is represented by the directed graph $G=(V, A)$, and the service curve of each node in the network is $\left\langle\beta_{v}, \varepsilon_{v}\right\rangle_{v \in V}$. The arrive curve of traffic $f$ is $\langle\alpha, \varepsilon\rangle$, the traffic $f$ enter the network in the node $v_{\text {in }}$, and leave the network in the node $v_{\text {out }}$. Now we find a path $p=\left(v_{0}, v_{1}, \ldots, v_{l}\right)$ for traffic $f$, in which $v_{0}=v_{\text {in }}, v_{l}=v_{\text {out }}$, and stochastic delay of the traffic $f$ in the path satisfies $\left\langle D_{\max }(f, p), \varepsilon_{d}\right\rangle$ of the stochastic backlog is $\left\langle B_{\max }(f, p), \varepsilon_{B}\right\rangle$.

According to [2], we need to know the arrive curve of $f$ and the service curve provided to the traffic $f$ by the network to get the stochastic delay $\left\langle D_{\max }(f, p), \varepsilon_{d}\right\rangle$. At present it is known that the arrive curve of traffic $f$ is $\langle\alpha, \varepsilon\rangle$, and yet we need to get the service curve provided to traffic $f$ by the network. We assume that the current traffic is independent of the cross traffic, the hypothesis lead to the process of getting the service curve provided to traffic $f$ by the network is becoming easy. According the left service curve thermo [3], the service provide to the traffic $f$ by the network is called left service curve, the gain of left service curve depends on the estimation of cross traffic. Comparing with the interdependent case of cross traffic, the estimation of left service curve is easy in the independent case of cross traffic. It is assumed that the route of traffic $f$ in the network is $p$ represented by dashed line, and the path $p_{i}$ represented by the solid line is route of cross traffic $f_{i}$. According to the left service curve theorem[3], the service curve provided to the traffic $f$ by the each node in 
the path $p$ is respectively $\beta_{\text {left }}^{1}=\beta_{1}-\alpha_{1}, \quad \beta_{\text {left }}^{4}=\beta_{4}, \quad \beta_{\text {left }}^{5}=\beta_{5}-\alpha_{2}, \quad \beta_{\text {left }}^{7}=\beta_{7}-\alpha_{3}$, and the error function is $\varepsilon$.According to the ( $\mathrm{min},+)$ convolution, the service curve provided to the traffic $f$ by the path $p$ is $\beta(p)=\beta_{1} \otimes \beta_{4} \otimes \ldots \otimes \beta_{7}$ without circle.

It is assumed that the arrive curve $E(t)=\alpha$ of traffic $f$ is convex, and the service curve $\beta_{i}$ in the network node is concave. And according to the Legend Transformation, the transformation of service curve provided to the traffic $f$ is

$$
\bar{L}_{E}(c, \alpha)=\sup _{t \geq 0}\{c t-\alpha\}
$$

For the $(\min ,+)$ function $x(t), y(t)$, their Legend Transformation is $L(x \otimes y)=L(x)+L(y)[4,5]$, the transformation of service curve provided by the path $p$ is

$$
\underline{L}_{S}(c, \beta)=\underline{L}_{S}\left(c, \beta_{1}\right)+\underline{L}_{S}\left(c, \beta_{4}\right)+\cdots+\underline{L}_{S}\left(c, \beta_{7}\right)
$$

\section{The QoS Route Optimization Algorithm Based on Legend Transformation}

According to [5], the delay and backlog parameter of the traffic $f$ is obtained through the path $p$. The step of QoS route optimization algorithm is proposed using formula (1) and formula (2), and process of the algorithm includes the following four step:

(1) Perform the Legend Transformation of service curve provided to the traffic $f$ by the path $p$ and Legend Transformation of arrive curve of the traffic $f$

(2) Calculate the service curve of path $p$ through by traffic $f$ according to formula (2)

(3) Calculate the stochastic delay or stochastic backlog according to [6]

(4) Compare the calculated QoS parameter with the demand of QoS parameter of traffic $f$, if the result meets the demand, the path can be the route of traffic $f$, if not, go to the step (1) and recalculate.

For the independent case of cross traffic, the end-to-end service curve have been given, and for the non-independent case of cross traffic, as [7] said, the method of adding node by node can't describe the PMOO phenomenon. Figure 5 describes the case of non-independent cross traffic. The cross traffic in the path of traffic $f$ is $f_{1}$ and $f_{2}$, and there is no cross path between traffic $f_{1}$ and $f_{2}$. Next we will give the calculation method of end-to-end service curve in the case of non-independent cross traffic in the condition of take account of the PMOO phenomenon.

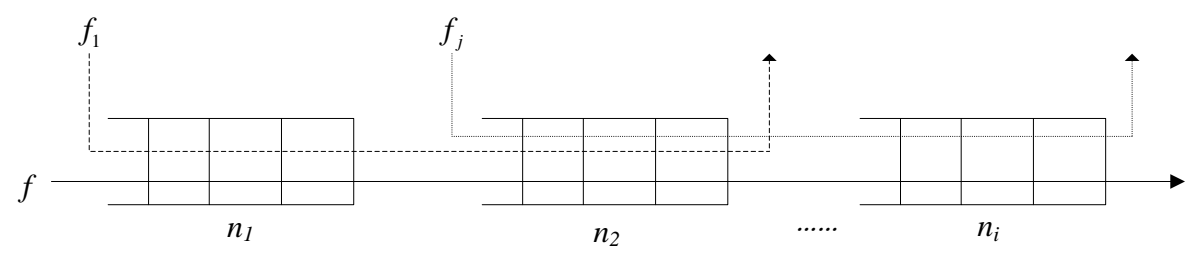

Figure 5. Network Model for Non-independent Cross Flow 
First, according to the left service curve theorem [3], the service curve provided to the traffic $f$ is calculated by node-one-node. In order to simplify the problem, assume there is only three nodes, and its service curve is respectively $\left\langle\beta_{i}, \varepsilon_{S}\right\rangle, i=1,2,3$, and the arrive curve of traffic $f_{1}$ and $f_{2}$ is respectively $\left\langle\alpha_{i}, \varepsilon_{E}\right\rangle$. According to the left service curve theorem [3], the service curve provided to traffic $f$ is

$$
\left\langle\left(\beta_{1}-\alpha_{1}\right) \otimes\left(\beta_{2}-\alpha_{1}-\alpha_{2}\right) \otimes\left(\beta_{3}-\alpha_{2}\right), \varepsilon_{E} \otimes \varepsilon_{S}\right\rangle
$$

Not taking account of PMOO, the service curve is given by Formula (3). To study the interaction of different traffics, it is assumed that the arrive function is concave, and service function is convex, Legend Transformation of formula (3) is performed to obtain the service curve in the case of PMOO, that is

$$
\begin{aligned}
& \underline{L}_{s}\left\{\left(\beta_{1}-\alpha_{1}\right) \otimes\left(\beta_{2}-\alpha_{1}-\alpha_{2}\right) \otimes\left(\beta_{3}-\alpha_{2}\right)\right\} \\
& =\underline{L}_{s}\left(\beta_{1}-\alpha_{1}\right)+\underline{L}_{s}\left(\beta_{2}-\alpha_{1}-\alpha_{2}\right)+\underline{L}_{s}\left(\beta_{3}-\alpha_{2}\right) \\
& =\inf _{t}\left\{\beta_{1}-\alpha_{1}-s t\right\}+\inf _{t}\left\{\beta_{2}-\alpha_{1}-\alpha_{2}-s t\right\}+\inf _{t}\left\{\beta_{3}-\alpha_{2}-s t\right\}
\end{aligned}
$$

According to inequality $\inf _{t}\{X(s)-Y(s)\} \geq \inf _{t} X(s)-\sup _{t} Y(s)$, formula (4) is rewritten as

$$
\begin{aligned}
& \underline{L}_{s}\left\{\left(\beta_{1}-\alpha_{1}\right) \otimes\left(\beta_{2}-\alpha_{1}-\alpha_{2}\right) \otimes\left(\beta_{3}-\alpha_{2}\right)\right\} \\
& \geq \underline{L}_{s}\left(\beta_{1}\right)+\underline{L}_{s}\left(\beta_{2}\right)+\underline{L}_{s}\left(\beta_{3}\right)-\left\{\sup _{t} \alpha_{1}+\sup _{t}\left(\alpha_{1}+\alpha_{2}\right)+\sup _{t} \alpha_{2}\right\} \\
& =\underline{L}_{s}\left(\beta_{1} \otimes \beta_{2} \otimes \beta_{3}\right)-\left\{\sup _{t} \alpha_{1}+\sup _{t}\left(\alpha_{1}+\alpha_{2}\right)+\sup _{t} \alpha_{2}\right\}
\end{aligned}
$$

\section{Numerical Analysis and Simulation}

The above section gives the calculation method of traffic QoS parameter in Legend domain, and the QoS route optimization algorithm is proposed based on the QoS parameter. The performance of the algorithm is decided by the accuracy of QoS parameter calculation. Therefore, first we give the QoS parameter of theoretic method and simulation method, analyze the accuracy of theory method, then compare the QoS route optimization algorithm presented by this paper and the other route algorithm. To verify the route algorithm presented by the paper, taking the service curve in the case of independent cross traffic as example, simulation is performed, and randomly select ten different arrive curves and a service curve, this result is shown in Figure 6. 


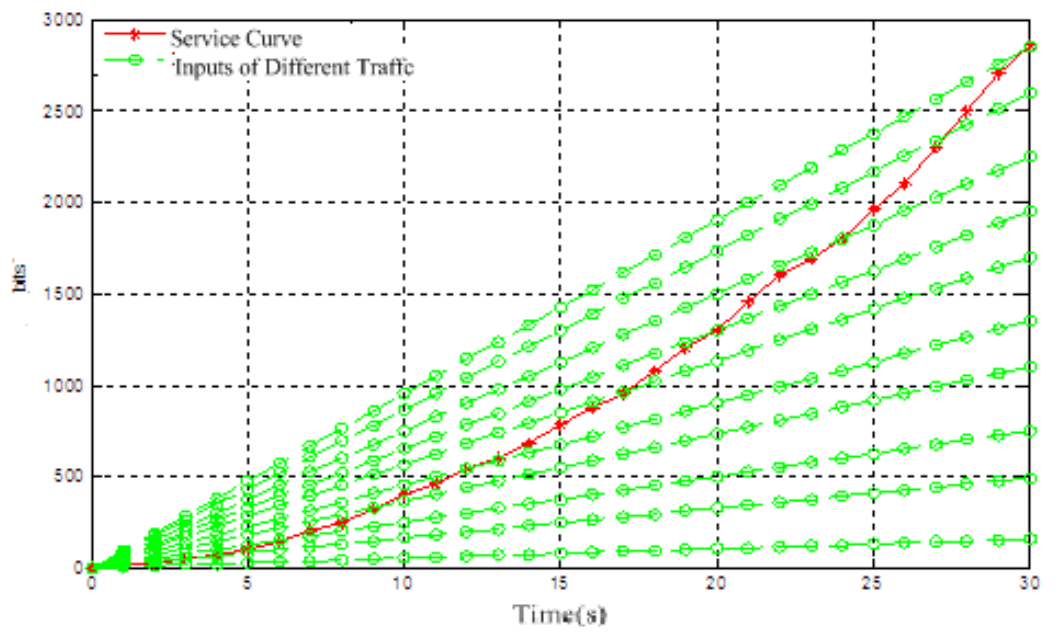

Figure 6. Input Traffic Flow and Service Curve in Simulation

In the circumstance of NS, the simulation is performed for the scene shown in figure 6 . By monitoring the output of network node, and combining the input of traffic, estimation of service in the Legend domain is obtained according to $H(\alpha)=L\{y(t)\}-L\{x(t)\}$, the simulation result is shown in figure 4. Here, $x(t)$ is input, $y(t)$ is output, and $L$ is Legend Transformation.

To verify that the theoretical model conform to the actual network situation, the result of theoretical analysis and the result of simulation is compared, the result is shown in figure 7. The comparison proves the service curve in the case of non-independent cross traffic adapts the actual network.

As the service curve is the foundation of QoS route optimization algorithm, the service curve shown in figure 7 can be used to calculate the QoS parameter accurately, and then select the route based on the calculation result, and the calculated service curve can adapt the actual network. Therefore, the validity of the algorithm is proved.

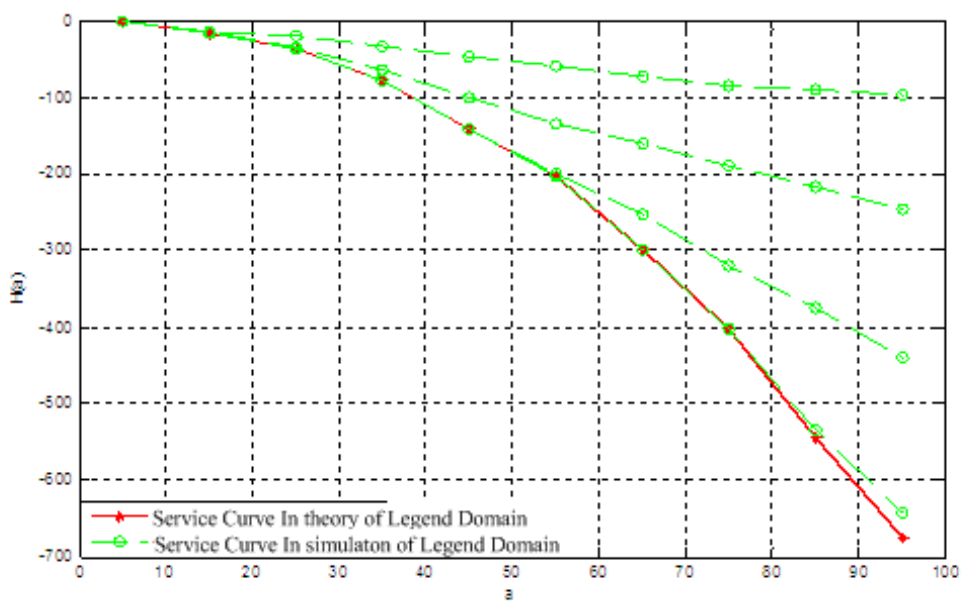

Figure 7. Comparison between Academic Legendre Service Curve and its Simulation 


\section{Conclusion}

For the case of independent cross traffic, transforming the convolution to the $(+,-)$ in legend domain reduce the complexity of service curve. Through performing the Legend Transformation of arrive curve and service curve, we can get the closed expression of stochastic delay and stochastic backlog. For the case of non-independent cross traffic, combining the left service curve and analyzing the service curve in Legend domain, we can get the cross relations of traffic in different path, and the expression of different traffic in condition of taking account the PMOO. Last, the simulation result proves the correctness the route optimization algorithm.

\section{Acknowledgements}

This work is supported by National Natural Science Foundation Major Research Plan of China (NSFC) (61301101).

\section{References}

[1] J.-Y. Le Boudec and P. Thiran, "Network calculus: a theory of deterministic queuing systems for the internet", Springer-Verlag, (2001).

[2] F. Ciucu, A. Burchard and J. Liebeherr, "A network service curve approach for the stochastic analysis of networks", IEEE/ACM Transactions on Networking, vol. 52, no. 6, (2006), pp. 2300$2312 \mathrm{P}$.

[3] A. Burchard, J. Liebeherr and S. D. Patek, "A min-plus calculus for end-to-end statistical service guarantees", IEEE Transactions on Communications, vol. 52, no. 9, (2006), pp. 4105-4114.

[4] M. Fidler and S. Recker, "Conjugate network calculus: a dual approach applying the Legendre transform”, Computer Networks, (2006), pp. 1026-1039.

[5] F. Agharebparast and V. C. M. Leung, "Slope domain modeling and analysis of data communication networks: a network calculus complement", IEEE International Conference on Communications, (2006), pp. 591-596.

[6] Y. Liu, C.-K. Tham and Yuming Jiang, "A calculus for stochastic Qos analysis", Performance Evaluation, vol. 64, no. 6, (2007), pp. 547-572.

[7] X. Huang and Y. Fang, "Multiconstrained QoS multipath routing in wireless sensor networks", Wireless Netw., vol. 14, (2008), pp. 465-478. 
International Journal of Future Generation Communication and Networking Vol. 7, No. 6 (2014) 